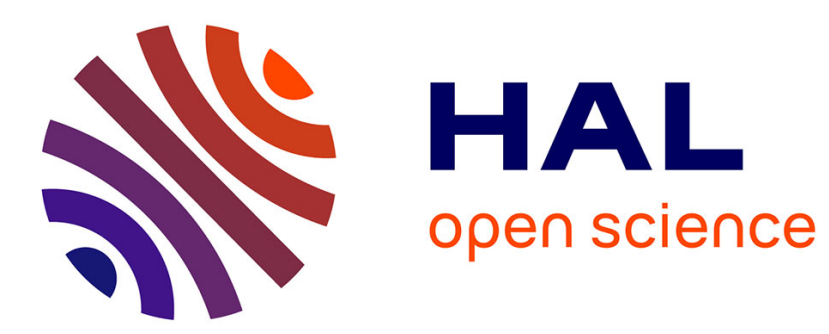

\title{
Derivation of supersaturation during precipitation from the mixing pattern of an inert tracer in the same device: case of partially premixed feed streams \\ R David, A Fall, Olivier Lecoq
}

\section{- To cite this version:}

R David, A Fall, Olivier Lecoq. Derivation of supersaturation during precipitation from the mixing pattern of an inert tracer in the same device: case of partially premixed feed streams. Chemical Engineering Science, 2003, 58 (22), pp.5079-5085. 10.1016/j.ces.2003.07.013 . hal-01717492

\author{
HAL Id: hal-01717492 \\ https://hal.science/hal-01717492
}

Submitted on 6 Nov 2019

HAL is a multi-disciplinary open access archive for the deposit and dissemination of scientific research documents, whether they are published or not. The documents may come from teaching and research institutions in France or abroad, or from public or private research centers.
L'archive ouverte pluridisciplinaire HAL, est destinée au dépôt et à la diffusion de documents scientifiques de niveau recherche, publiés ou non, émanant des établissements d'enseignement et de recherche français ou étrangers, des laboratoires publics ou privés. 


\title{
Derivation of supersaturation during precipitation from the mixing pattern of an inert tracer in the same device: case of partially premixed feed streams
}

\author{
René David*, Ahmed Fall, Olivier Lecoq \\ Laboratoire de Génie des Procédés des Solides Divisés, EMAC-CNRS, 81013 Albi Cedex9, France
}

\begin{abstract}
The average supersaturation field is predicted for precipitations in the case of partially premixed feed streams by a simple mixing model from a couple of successive experiments with the same inert tracer by Planar Laser Induced Fluorescence and using a single camera. Then, the mathematical derivation is different from that one used for the unmixed feed case. The corresponding experiments have been achieved in a $90^{\circ}$ impinging jets premixer and the supersaturation field was predicted. Due to the non-simultaneity of tracer experiments, a calculation of the averaged nucleation flux is not possible in the premixed feed case. Nevertheless, a comparison between different premixers can be done on the basis of the generated supersaturation levels and fouling risks.
\end{abstract}

Keywords: Mixing; Precipitation; PLIF; Nucleation; Tracer; Supersaturation

\section{Introduction}

Basically, precipitation is a chemical reaction between $\mathrm{A}$ and $\mathrm{B}$, yielding a non-soluble particulate precipitate $\mathrm{S}$ :

$n \mathrm{~A}+m \mathrm{~B} \rightarrow \mathrm{S}$ (solid),

where $n$ and $m$ generally lie between 1 and 3 .

Precipitation encompasses the so-called primary nucleation step, which generates the nuclei from the liquid phase and further steps forming the particle like growth, agglomeration, breakage or secondary nucleation, which generates additional nuclei from existing particles. Precipitations are generally characterized by small particles and short to medium precipitation times (Mersmann, 2001). According to the length and time scales defined by Villermaux and David (1988), this corresponds to high nucleation and medium growth rates.

Premixing is supposed to generate higher local supersaturation consecutive to contacting between highly concentrated fresh reagents from the feed streams. Thus, higher nucleation rates and in turn smaller particles are produced

\footnotetext{
* Corresponding author. Tel.: +33-563493236; fax: +33-563-49-3025.

E-mail address: rdavid@enstimac.fr (R. David).
}

(see for instance the pioneering work of Tosun (1988)). Baldyga, Podgorska, and Pohorecki (1995) and Baldyga and Bourne (1999) presented several mixing models for precipitations in tubes and stirred tanks with partial premixing based on a complete description of the mixing process. The fit with the corresponding experimental results on barium sulphate was generally fair and the influence of operating parameters correctly predicted, but their velocity and concentration fields were computed. Therefore, their models are relatively expensive in both computation time and effort. Zauner and Jones (2002) tried an other approach by using the segregated feed model (SFM) of Villermaux (1989), where the entering fluids undergo macro- and meso-mixing together and with the rest of the tank bulk, applied to double jet semi-batch precipitation of calcium oxalate.

In recent papers, a simulation of the initial mixing of two separated feed jets of reactive solutions in virtual ionic precipitation was presented (Fall, Lecoq, \& David, 2002; David, Fall, \& Lecoq, 2003). The mixed volume was split into fluid fractions originating from the jet and from the bulk. The paper demonstrates the possibility to predict the supersaturation and the nucleation flux fields in a mixed precipitator from concentration measurements of an inert tracer in the same precipitator geometry. One may try to implement the same procedure described in David et al. (2003) in the case 
of partially premixed feed streams. However, impossibility arises when doing so because the procedure requires the identification in the mixing plume of the molecules coming from every feed stream and from the rest of the tank: in other words, a given tracer concentration can be achieved by different combinations of portions of fluids coming from these three environments.

Fox (1998) did a somewhat similar approach of fluid mixing relying on the general mixing model (GMM), introduced by Villermaux and Falk (1994). He distinguished 4 different modes respectively (feed 1, feed 2 and two levels of mixed bulk). Each spatial point is characterized by a probability to be in one of these modes. Piton, Fox, and Marcant (2000) applied it to the precipitation of barium sulphate in a tubular precipitator. As they intended to calculate the concentration of species and the complete crystal size distributions of the precipitate, they took also nucleation and growth rates into account. Marchisio, Barresi, and Garbero (2002) simplified it to three modes (feed 1, feed 2 and one single mixed bulk) and calculated the moments of the CSD taking into account the presence of agglomeration. Nevertheless, their model and calculations are rather difficult to handle.

The present paper introduces a mixing model in the case of partially premixed feed streams, in order to keep the simplicity of our previous approach and to overcome the difficulty of dealing with three origins of fluids (or modes).

\section{Experimental apparatus}

The planar laser induced fluorescence (PLIF) Technique and the experimental apparatus used in this work are described and available in Fall, Lecoq, and David (2001). The mixing device, which is tested here, is a standard tank equipped with a Rushton turbine and two $90^{\circ}$-impinging jets mixer. Mahajan and Kirwan (1996) and Benet, Muhr, Plasari, and Rousseaux (2002) have extensively studied such impinging mixers. However, both groups of authors used an angle equal to or close to $180^{\circ}$ between the jets (opposite jets). During tracer experiments, the impinging jet mixer feeds fresh tracer solution and pure solvent. The feed point locations are shown in Fig. 1. The stirring speed is $N=3 \mathrm{~s}^{-1}$ and the flow rates $Q_{L}=Q_{R}=1.35 \mathrm{dm}^{3} \mathrm{~min}^{-1}$.

\section{Model}

Imagine the situation depicted by Fig. 2, where the two feed stream jets are partially premixed $\left(90^{\circ}\right.$-impinging jets $)$. It would be interesting to predict the supersaturation field from inert tracer concentration measurements in the same device.

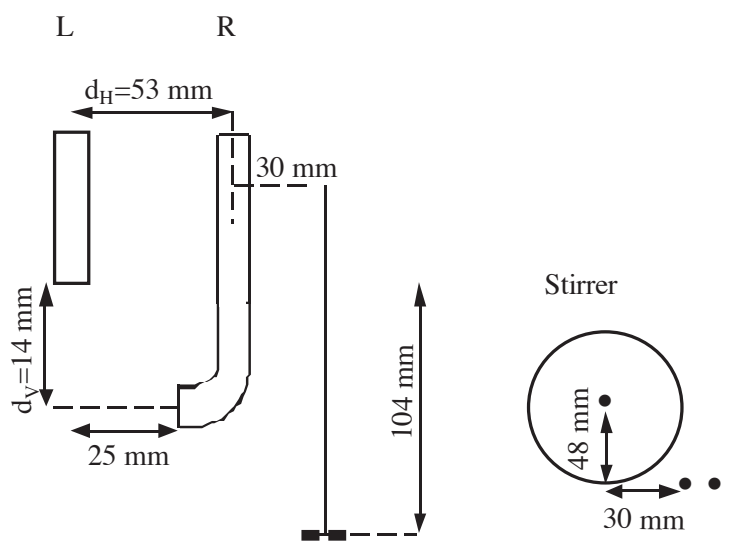

Fig. 1. Experimental set-up. Left: relative position of fed streams L and $\mathrm{R}$; right: view from above; locations of both feed streams with respect to the stirrer.

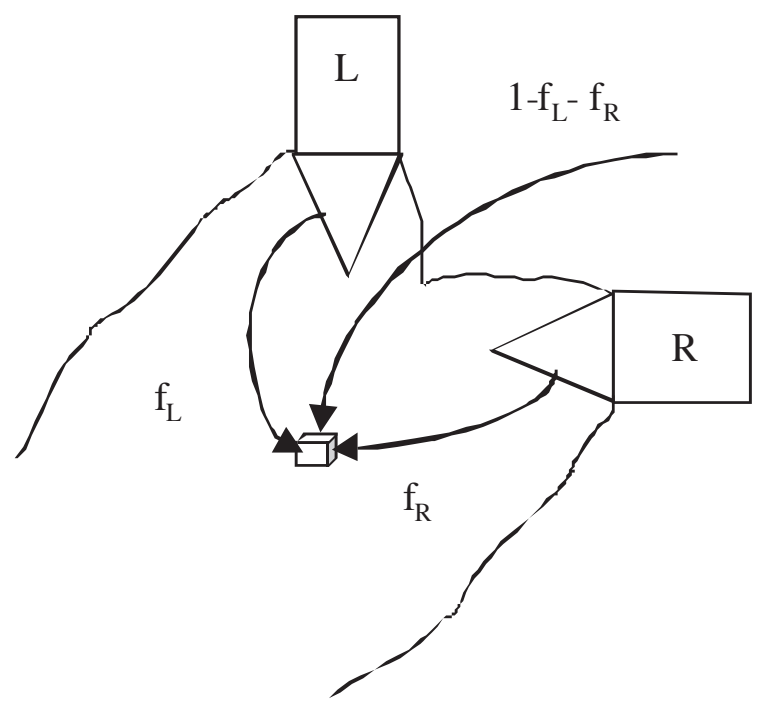

Fig. 2. Partially premixed feed streams model.

Let us consider two mixing experiments with tracers, which denote $E_{1}$ and $E_{2}$ (Fig. 3). In $E_{1}$, the inert tracer is injected in the right feed streams with flow rate $Q_{R}$. A third inlet feed stream with flow rate $Q_{3}$ is located far from the two others. The mixing zone of both fresh fluid feed streams is described by two fractions $f_{R}$ and $f_{L}$, which we denote the fluid fractions at point $(x, y, z)$ and time $t$, and which originate from feed tubes $\mathrm{R}$ and $\mathrm{L}$, respectively. Each elementary volume of this zone is made of fractions $f_{R}$ and $f_{L}$ of incompressible fluid coming from the incoming feed streams and of the complementary fraction $1-f_{L}-f_{R}$, coming from the bulk where uniform concentration $C_{T_{0}}$ is assumed. Note that experiments $E_{1}$ and $E_{2}$ can be performed at different times with the same or different tracers, provided that the linearity of signal against tracer concentration is verified. 

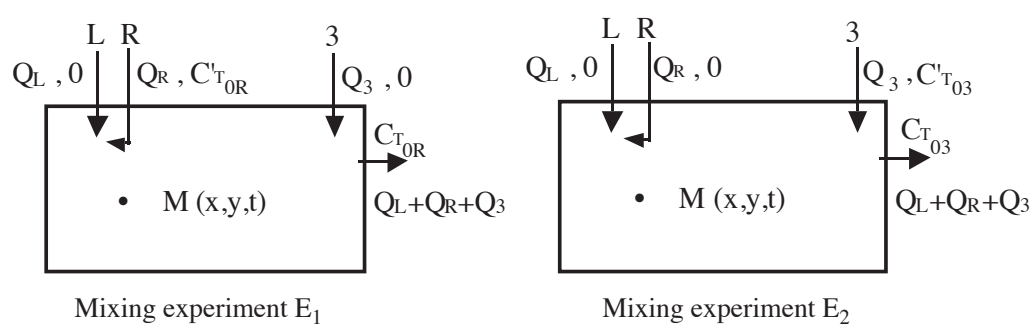

Fig. 3. Mixing of two partially premixed jets of inert tracers.

The relation between the inlet and outlet concentration can be written

$C_{T_{0} R}^{\prime}=C_{T_{0} R}\left(1+\frac{Q_{L}}{Q_{R}}+\frac{Q_{3}}{Q_{R}}\right)=C_{T_{0} R}\left(1+\frac{1+v_{3}}{v}\right)$,
$C_{T_{0} 3}^{\prime}=C_{T_{0} 3}\left(1+\frac{Q_{R}}{Q_{3}}+\frac{Q_{L}}{Q_{3}}\right)=C_{T_{0} 3}\left(1+\frac{1+v}{v_{3}}\right)$

with

$v=Q_{R} / Q_{L} \quad$ and $\quad v_{3}=Q_{3} / Q_{L}$.

At a given point $\mathrm{M}$, if we could monitor $C_{T R}$ and $C_{T 3}$ simultaneously, we could write

$C_{T R}=\left(1-f_{L}-f_{R}\right) C_{T_{0} R}+C_{T_{0} R}^{\prime} f_{R}$,

$C_{T 3}=\left(1-f_{L}-f_{R}\right) C_{T_{0} 3}$

which yields

$\frac{C_{T R}}{C_{T_{0} R}}=X_{T R}=1-f_{L}+\left(\frac{1+v_{3}}{v}\right) f_{R}$

and

$\frac{C_{T 3}}{C_{T_{0} 3}}=X_{T 3}=1-f_{L}-f_{R}$.

When $f_{L}$ and $f_{R}$ are substituted in the above equation, we obtained

$f_{R}=\frac{X_{T R}-X_{T 3}}{1+\left(1+v_{3}\right) / v}$,

$f_{L}=1-\frac{X_{T R}+\left(\left(1+v_{3}\right) / v\right) X_{T 3}}{1+\left(\left(1+v_{3}\right) / v\right)}$.

Let us consider now a virtual reactive experiment, which we denote $\mathrm{E}_{3}$ and corresponding to real ionic precipitation (Fig. 4).

The simplified reaction scheme is given by

$\mathrm{A}+\mathrm{B} \rightarrow \mathrm{S} \quad$ with $m=n=1$.

At the same point $(x, y, z)$ in the tank we assume that there is no change in hydrodynamics between experiments $E_{1}, E_{2}$ and $E_{3}$. If we had performed $E_{3}$ instead of $E_{1}, E_{2}$, at the same time and location, the concentrations of reagents $\mathrm{A}$
L R 3

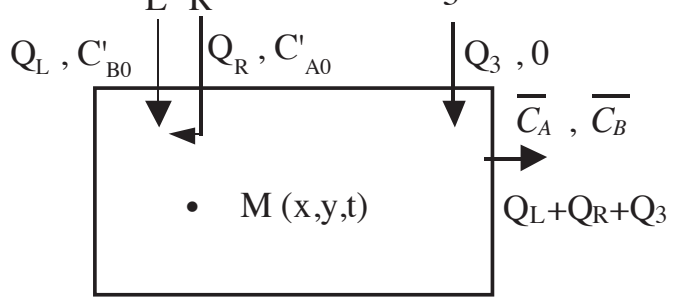

Virtual mixing experiment $\mathrm{E}_{3}$

Fig. 4. Mixing of two partially premixed jets of reactants.

and $\mathrm{B}$ would be respectively

$C_{A}=\left(1-f_{L}-f_{R}\right) \bar{C}_{A}+C_{A 0}^{\prime} f_{R}$,

$C_{B}=\left(1-f_{L}-f_{R}\right) \bar{C}_{B}+C_{B 0}^{\prime} f_{L}$.

The relative supersaturation is defined by $S=C_{A} C_{B} / P_{S}-1$ (for $m=n=1$ ), and is expressed by

$$
\begin{aligned}
S= & \left(1-f_{R}-f_{L}\right)^{2} \frac{\bar{C}_{A} \bar{C}_{B}}{P_{S}}-1 \\
& +\frac{C_{A 0}^{\prime} \bar{C}_{B}}{P_{S}}\left(f_{R}-f_{R}^{2}-f_{R} f_{L}\right) \\
& +\frac{C_{B 0}^{\prime} \bar{C}_{A}}{P_{S}}\left(f_{L}-f_{L}^{2}-f_{R} f_{L}\right) \\
& +f_{R} f_{L} \frac{C_{A 0}^{\prime} C_{B 0}^{\prime}}{P_{S}} .
\end{aligned}
$$

If feed is stoichiometric

$\bar{C}_{A}=\bar{C}_{B} \quad$ and $\quad \frac{C_{B 0}^{\prime}}{C_{A 0}^{\prime}}=\frac{Q_{R}}{Q_{L}}=v$.

Then the supersaturation becomes

$$
\begin{aligned}
S= & \frac{\bar{C}_{A} \bar{C}_{B}}{P_{S}}\left(1+f_{R}^{2}+f_{L}^{2}-2 f_{R}-2 f_{L}-2 f_{R} f_{L}\right)-1 \\
& +\frac{C_{A 0}^{\prime} \overline{C_{A}}}{P_{S}}\left(f_{R}-f_{R}^{2}-f_{R} f_{L}+v f_{L}-v f_{L}^{2}-v f_{R} f_{L}\right) \\
& +\frac{C_{A 0}^{\prime 2}}{P_{S}} v f_{L} f_{R} .
\end{aligned}
$$


We have chosen $Q_{L}=Q_{R}(v=1)$ and the feed stream $Q_{3}$ is very low compared to the others $\left(v_{3}=0\right)$ - but the tracer solution remains concentrated in order to be detectableEqs. (9) and (10) are considerably simplified

$f_{R}=\frac{X_{T R}-X_{T 3}}{2}$,

$f_{L}=1-\left(\frac{X_{T R}+X_{T 3}}{2}\right)$.

Assuming $\bar{C}_{A}$ and $\bar{C}_{B}$ to be close to equilibrium in the bulk of the tank (see David et al., 2003), the supersaturation defined by Eq. (13) becomes

$$
\begin{aligned}
S= & \left(-1-2 X_{T R}+2 X_{T 3}+X_{T R}^{2}\right)+\frac{C_{A 0}^{\prime}}{\sqrt{P_{S}}}\left(X_{T 3}-X_{T 3}^{2}\right) \\
& +\frac{C_{A 0}^{\prime 2}}{P_{S}}\left(\frac{X_{T R}-X_{T 3}}{2}+\frac{X_{T 3}^{2}-X_{T R}^{2}}{4}\right) .
\end{aligned}
$$

Taking into account $C_{A 0}^{\prime} \gg \sqrt{P_{s}}$, we can neglect the first term of Eq. (18).

And, finally, the supersaturation is expressed by

$$
\begin{aligned}
S= & \frac{C_{A 0}^{\prime}}{\sqrt{P_{S}}}\left(X_{T 3}-X_{T 3}^{2}\right) \\
& +\frac{C_{A 0}^{\prime 2}}{2 P_{S}}\left(X_{T R}-X_{T 3}+\frac{X_{T 3}^{2}-X_{T R}^{2}}{2}\right) .
\end{aligned}
$$

The first term of the right-hand side of Eq. (19) corresponds to the contribution of the mixing between fresh fluids and the bulk, whereas the second one is related to the direct mixing of the entering fresh fluids. From the instantaneous supersaturation, it is easy to derive the average supersaturation by

$$
\begin{aligned}
\bar{S}= & \frac{C_{A 0}^{\prime}}{\sqrt{P_{S}}}\left(\overline{X_{T 3}}-\overline{X_{T 3}^{2}}\right) \\
& +\frac{C_{A 0}^{\prime 2}}{2 P_{S}}\left(\overline{X_{T R}}-\overline{X_{T 3}}+\frac{\overline{X_{T 3}^{2}}-\overline{X_{T R}^{2}}}{2}\right) .
\end{aligned}
$$

It is worthwhile noting that expression (20) contains no cross terms $\overline{X_{T R} X_{T 3}}$. From Eqs. (9), (10) and (15), it can be demonstrated that this holds if, in the general case $(m=n=1)$

$Q_{R}=Q_{L}+Q_{3} \quad$ i.e. $v=1+v_{3}$.

It is then possible to monitor and average $X_{T R}$ and $X_{T 3}$ separately for the calculation of $\bar{S}$ by Eq. (20). The average supersaturation can be reduced by $S_{\max }=C_{A 0}^{\prime 2} / 4 P_{S}$. $S_{\max }$ represents the highest achievable value of $\bar{S}$ when premixing both feed streams. Generally, the second term of the summation at the right hand side of Eq. (20) is larger than the first one, because of the experimentally high values of $C_{A 0}^{\prime} / \sqrt{P_{s}}$. If no interaction would exist between the feed streams and the rest of the tank (pure premixing), $X_{T 3}$ would be nil. Then Eq. (20) reduces to

$\bar{S}=\frac{C_{A 0}^{\prime 2}}{2 P_{S}}\left(\overline{X_{T R}}-\frac{\overline{X_{T R}^{2}}}{2}\right)$.
The next step would consist of the calculation of the nucleation fluxes. Nucleation rate, as well homogeneous as heterogeneous nucleation, is generally expressed by the Volmer and Weber (1926) relationship:

$R_{N}=A_{N} \exp \left[\frac{-B_{N}}{\log _{e}^{2} S}\right]$.

The average reduced nucleation flux is expressed by

$$
\begin{aligned}
\overline{\frac{R_{N}}{A_{N}}}= & \overline{\exp \left[-\frac{B_{N}}{\log _{e}^{2} S}\right]} \\
= & \int_{X_{T R}=0}^{2} \int_{X_{T 3}=0}^{2} \exp \left(\frac{-B_{N}}{\log _{e}^{2} S\left(X_{T R}, X_{T 3}\right)}\right) \\
& \times p\left(X_{T R}, X_{T 3}\right) \mathrm{d} X_{T 3} \mathrm{~d} X_{T R} .
\end{aligned}
$$

However, the demonstration made above for the average supersaturation cannot be applied to relation (24). Actually, the calculation of $\overline{R_{N}} / A_{N}$ imposes the knowledge of the probability $p\left(X_{T R}, X_{T 3}\right)$ to have simultaneously the values $X_{T R}$ and $X_{T 3}$ at the same point. This would require that $X_{T R}$ and $X_{T 3}$ are simultaneously measured at this point, and, consequently, it would necessitate two different tracers and two monitoring cameras.

\section{Experimental results with an inert tracer}

The average reduced concentration is shown in Fig. 5 for the $90^{\circ}$-impinging jets mixing. In the Fig. 5(b), the two perpendicular feed pipes contain pure water and the reduced tracer concentration is $X_{T 3}$, whereas, in the Fig. 5(a) one of the two perpendiculars feed pipes contains the solution of inert tracer and the other one contains the pure water. In this case the reduced tracer concentration is $X_{T R}$.

The reduced tracer concentration varies between 0 (black), in the pure water feed stream jets, to 2 (white), in the inert tracer feed stream jets. A pixel represents a square of an area about $70 \times 70 \mu \mathrm{m}^{2}$ in Figs. 5( $\mathrm{a}$ and $\left.\mathrm{b}\right)$.

\section{The virtual experimental results with an in ionic precipitation}

By applying Eq. (20) to the concentrations fields resulting from experiments $E_{1}$ and $E_{2}$ we can predict the average supersaturation field for every pixel. The result is given in Fig. 6. High local supersaturations induce high local nucleation rates (see Eq. (23)). However, when applying Eq. (24), one should remark that $R_{N}$ is a non-linear function of $S$ and thus

$\overline{R_{N}(S)} \neq R_{N}(\bar{S})$.

A highly supersaturated zone appears in the mixing layer between the impinging jets with a maximum value of 0.72 for the reduced average supersaturation, which is relatively high with respect to the maximum value of 1 . A more detailed analysis is made in Fig. 7: The reduced supersatura- 


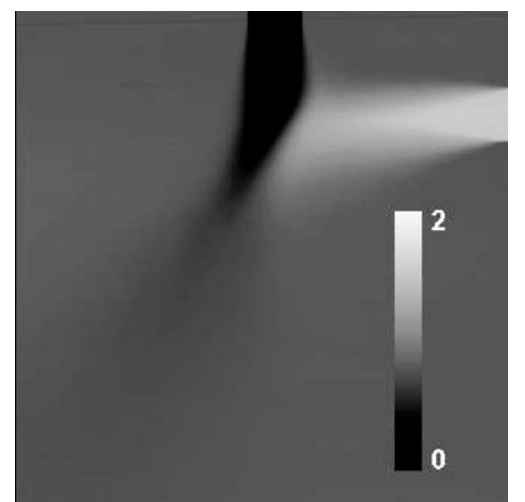

(a) $\quad X_{T R}\left(\right.$ experiment $\left.\mathrm{E}_{1}\right)$

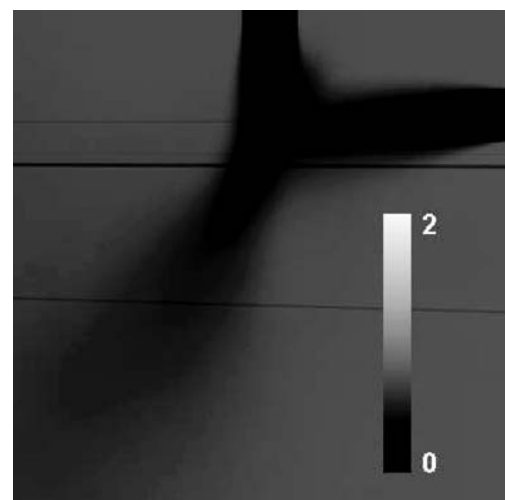

(b) $\quad X_{T 3}\left(\right.$ experiment $\left.\mathrm{E}_{2}\right)$

Fig. 5. Average reduced tracer concentrations: (a) the tracer is introduced in the right (horizontal) feed stream; (b) the tracer is introduced via feed 3.

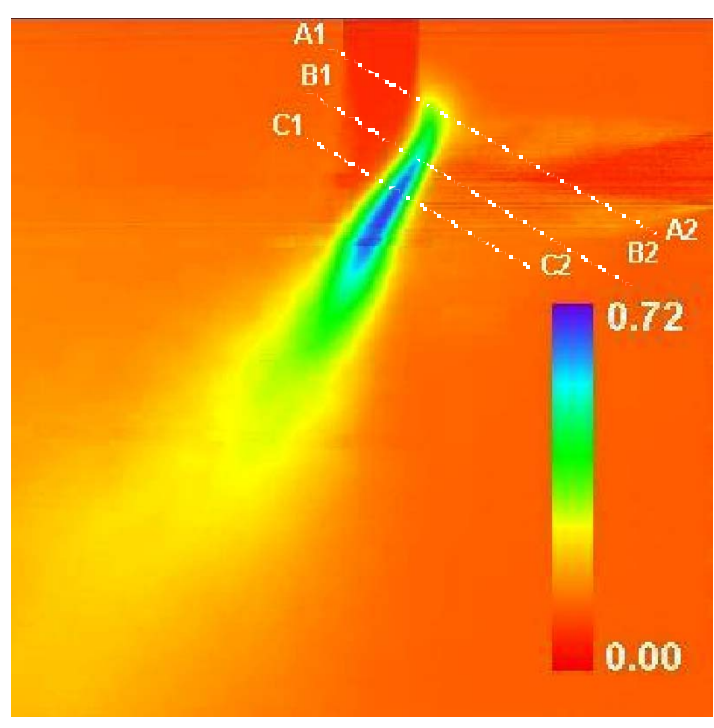

Fig. 6. Average reduced supersaturation $\bar{S} / S_{\max }$.

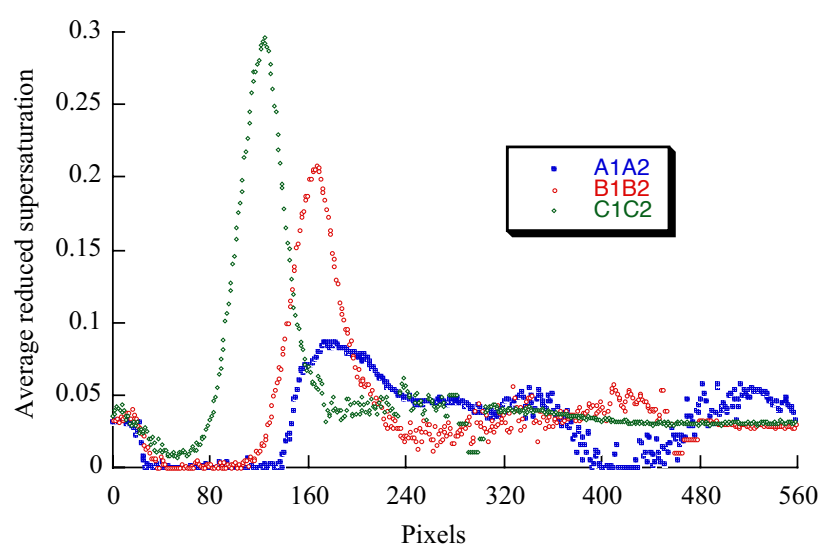

Fig. 7. Profiles of average reduced supersaturation $\bar{S} / S_{\max }$ along the lines $\mathrm{A} 1 \mathrm{~A} 2, \mathrm{~B} 1 \mathrm{~B} 2, \mathrm{C} 1 \mathrm{C} 2$ defined in Fig. 6. tion profiles on straight lines A1A2, B1B2 and C1C2 are plotted. Zero supersaturations are observed in the core of the jets on each line. The mixing plume shows average values about 0.05 , while the highest peaks (up to 0.3 on line $\mathrm{C} 1 \mathrm{C} 2$ ) are noticed in the central part of the mixing layer.

In this configuration, the risk of fouling on the inlet tubes is low, because the highest supersaturations arise far from the tubes.

\section{Discussion}

The same discussion on time and space resolution as for unmixed feed streams (David et al., 2003) still applies. During $35 \mathrm{~ms}$ exposure time, with an order of magnitude of the velocity of $0.1 \mathrm{~m} \mathrm{~s}^{-1}$ as expected in the jets, an eddy moves on about 50 pixels in the main flow direction. Consequently, exposure times of each pixel under $1 \mathrm{~ms}$ are recommended. Even in this case, the spatial resolution will not be better than the size of one pixel, i.e. $70 \mu \mathrm{m}$. A better resolution in exposure time of the tracer images would increase the averaged squared terms $\overline{X_{T R}^{2}}$ and $\overline{X_{T 3}^{2}}$ in Eqs. (20) and (22). Especially $\overline{X_{T R}^{2}}$ would be modified because $X_{T R}$ has a wider range of variation than $X_{T 3}$ (see Fig. 5). Thus the calculated average supersaturation would be reduced.

As noted in the preceding paper (David et al., 2003), the apparent paradox is that the average supersaturations are decreasing functions of the variance $\sigma_{T R}^{2}$ :

$\bar{S}=\frac{C_{A 0}^{\prime 2}}{2 P_{S}}\left(\overline{X_{T R}}-\overline{X_{T 3}}+\frac{\sigma_{T 3}^{2}+{\overline{X_{T 3}}}^{2}-\sigma_{T R}^{2}-{\overline{X_{T R}}}^{2}}{2}\right)$.

Again, Eq. (26) indicates that the highest average supersaturations are obtained at locations where (a) $\overline{X_{T R}} \neq 2$ and 
$\overline{X_{T 3}} \neq 0$, or (b) both $\overline{X_{T R}}$ and $\overline{X_{T 3}} \neq 1$ and (c) variance $\sigma_{T R}^{2}$ is small (i.e. negligible fluctuations of tracer concentrations during experiment $E_{1}$ ). Indeed, such locations are grossly the same as those for non-zero variance, but the areas of maximum supersaturations differ from those for maximum variances.

Generally, the goal of such premixing devices is to produce very small particles. As a consequence of the threshold effect illustrated by Eqs. (23), (24), the actual supersaturation must be as high as possible in a wide premixing zone in order to maximize the nuclei production flux. This is likely to occur when the average supersaturation is high over a large area. It is thus possible to detect premixing devices with a high ability to produce very small particles.

\section{Conclusion}

Averaged supersaturation fields in partially premixed feed precipitators were predicted from tracer concentration images obtained by PLIF by a simple mixing model. The method is based on the experimental monitoring of the same area during two successive experiments, where the tracer is injected via either one of the feed streams or a third additional feed stream with very a low flow rate. Thus, the most supersaturated regions could be determined for two feed streams impinging at $90^{\circ}$. A quantitative comparison of different mixing devices is thus possible, provided that instantaneous tracer concentration images are available. Zones or points with higher fouling risks can be foreseen, in order to avoid non-adapted geometries when performing precipitations with frequent occurrence of scaling.

However, due to the non-simultaneity of both records, the method was not able to also predict the averaged nucleation flux as it was previously done in the case of unmixed feed precipitators. The simultaneous use of two different tracers should make it possible to overcome this difficulty.

Other improvements of the method would consist of the reduction of exposure time in order to monitor faster fluctuations (i.e. smaller scales of mixing).

\section{Notation}
$A_{N} \quad$ constant, Eq. (23), $\mathrm{m}^{-3} \mathrm{~s}^{-1}$
$B_{N} \quad$ constant, Eq. (23), dimensionless
$C \quad$ concentration, $\mathrm{mol} \mathrm{m} \mathrm{m}^{-3}$
$\overline{C_{A}} \quad$ mixing concentration of reagent $\mathrm{A}$ in the out- let, $\mathrm{mol} \mathrm{m}^{-3}$
$C_{A 0}^{\prime} \quad$ feed concentration of reagent $\mathrm{A}, \mathrm{mol} \mathrm{m}^{-3}$
$\frac{C_{A}}{C_{B}}$
$C_{B 0}^{\prime} \quad$ feed concentration of reagent $\mathrm{B}, \mathrm{mol} \mathrm{m}^{-3}$
$C_{T 0} \quad$ mixing tracer concentration in the outlet, $\mathrm{mol} \mathrm{m}^{-3}$

$\begin{array}{ll}C_{T 0}^{\prime} & \begin{array}{l}\text { feed concentration of fluorescent tracer, } \\ \text { mol } \mathrm{m}^{-3}\end{array} \\ f_{L} & \begin{array}{l}\text { feed stream fraction coming from the left in- } \\ \text { let, dimensionless } \\ \text { feed stream fraction coming from the right } \\ \text { inlet, dimensionless }\end{array} \\ f_{R} & \text { height of the liquid in the tank, } \mathrm{m} \\ H & \text { stirring speed, } \mathrm{s}^{-1} \\ N & \text { joint probability density function, dimension- } \\ p & \text { less } \\ P_{S} & \text { solubility product, mol } \mathrm{m}^{-6} \\ Q & \text { feed flow rate, } \mathrm{dm}^{3} \mathrm{~s}^{-1} \\ R_{N} & \text { nucleation flux, } \mathrm{m}^{-3} \mathrm{~s}^{-1} \\ S & \text { supersaturation, dimensionless } \\ S_{\mathrm{max}}=\frac{C_{A 0}^{\prime 2}}{4 P_{S}} & \text { maximum achievable value of } \bar{S}, \text { dimension- } \\ & \text { less } \\ T & \text { internal diameter of the tank, } \mathrm{m} \\ v & \text { flow rate ratio, dimensionless } \\ V & \text { volume, m }\end{array}$

\section{Subscripts}

A

B

$\mathrm{L}$

$\mathrm{R}$

$\mathrm{T}$

third feed stream (in the bulk of the tank far away from the feed streams $\mathrm{L}$ and $\mathrm{R}$ )

reactant $\mathrm{A}$

reactant $B$

left

right

Superscripts

$\begin{array}{ll}m, n & \text { stoichiometric coefficients, dimensionless } \\ - & \text { time average }\end{array}$

\section{References}

Baldyga, J., \& Bourne, J. R. (1999). Turbulent mixing and chemical reactions (pp. 810-840). Chichester-New York-Weinheim-BrisbaneSingapore-Toronto: Wiley.

Baldyga, J., Podgorska, W., \& Pohorecki, R. (1995). Mixing-precipitation model with application to double-feed semibatch precipitation. Chemical Engineering Science, 50(8), 1281-1300.

Benet, N., Muhr, H., Plasari, E., \& Rousseaux, J. M. (2002). New technologies for the precipitation of solid particles with controlled properties. Powder Technology, 128(2-3), 93-98.

David, R., Fall, A., Lecoq, O. (2003). Derivation of supersaturation and nucleation flux during precipitation from the mixing pattern of an inert tracer in the same device: Case of unmixed feed streams, Chemical Engineering Science, 58(13), 2883-2891.

Fall, A., Lecoq, O., \& David, R. (2001). Characterization of mixing in a stirred tank by planar laser induced fluorescence (PLIF). Trans IchemE, 79(A), 876-882.

Fall, A., Lecoq, O., \& David, R. (2002). Derivation of supersaturation and nucleation flux in a stirred tank from tracer concentration measurements 
by P.L.I.F. 15th international symposium on industrial crystallization, Sorrento, Italy.

Fox, R. O. (1998). On the relationship between Lagrangian micromixing models and computational fluid dynamics. Chemical Engineering Proceeding, 37(6), 521-535.

Mahajan, A., \& Kirwan, D. J. (1996). Micromixing effects in a two-impinging jets precipitator. A.I.Ch.E. Journal, 42(7), 1801-1814.

Marchisio, D. L., Barresi, A. A., \& Garbero, M. (2002). Nucleation, growth and agglomeration in barium sulphate turbulent precipitation. A.I.Ch.E. Journal, 48(9), 2039-2050.

Mersmann, A. (2001). Crystallization technology handbook (2nd ed.) (pp. 45-79). New York: Marcel Dekker.

Piton, D., Fox, R. O., \& Marcant, B. (2000). Simulation of fine particle formation by precipitation using computational fluid dynamics. Canadian Journal of Chemical Engineering, 78, 983-993.
Tosun, G. (1988). Effect of addition mode and intensity on particle size distribution in barium sulphate precipitation, Proceedings of the 6th European conference on mixing, Pavia, Italy (pp. 161-170).

Villermaux, J. (1989). A simple model for partial segregation in a semibatch reactor. AIChE annual meeting, San Francisco, paper 114a.

Villermaux, J., \& David, R. (1988). Effet du micromélange sur la précipitation. Journal de Chimie Physique, 85, 273-279.

Villermaux, J., \& Falk, L. (1994). A generalized mixing model for initial contacting of reactive fluids. Chemical Engineering Science, 49, $5127-5140$.

Volmer, M., \& Weber, A. (1926). Keimbildung in übersättigten Gebilden. Zeitschrift fuer Physikalische Chemie, 119, 277-301.

Zauner, R., \& Jones, A. G. (2002). On the influence of mixing on crystal precipitation processes - application of the segregated feed model. Chemical Engineering Science, 57, 821-831. 